N. Toda

Nagoya Math. J.

Vol. 66 (1977), 37-52

\title{
SUR QUELQUES COMBINAISONS LINÉAIRES \\ EXCEPTIONNELLES AU SENS \\ DE NEVANLINNA, $V$.
}

NOBUSHIGE TODA

\section{Introduction}

Soit $f=\left(f_{0}, f_{1}, \cdots, f_{n}\right)(n \geqq 1)$ un système transcendant dans le plan $|z|<\infty$; c'est-à-dire, les fonctions $f_{0}, \cdots, f_{n}$ sont entières sans zéros communs à toutes et

$$
\lim _{r \rightarrow \infty} \frac{T(r, f)}{\log r}=\infty
$$

où $T(r, f)$ est la fonction caractéristique définie par Cartan ([1]) :

$$
T(r, f)=\frac{1}{2 \pi} \int_{0}^{2 \pi} \max _{0 \leqq j \leqq n} \log \left|f_{j}\left(r e^{i \theta}\right)\right| d \theta-\max _{0 \leqq j \leqq n} \log \left|f_{j}(0)\right| .
$$

Soit

$$
F=a_{0} f_{0}+\cdots+a_{n} f_{n} \quad(\not \equiv 0)
$$

une combinaison linéaire de $f_{0}, \cdots, f_{n}$, homogène à coefficients constants. On dit que la combinaison $F$ est

1) lacunaire si elle n'admet pas de zéro dans $|z|<\infty$;

2) exceptionnelle au sens de Picard si elle n'admet qu'un nombre fini de zéros dans $|z|<\infty$;

3) exceptionnelle au sens de Nevanlinna si

$$
\delta(F)=1-\limsup _{r \rightarrow \infty} \frac{N(r, 0, F)}{T(r, f)}>0 .
$$

On note que 1) $\Rightarrow 2) \Rightarrow 3$ ) et $0 \leqq \delta(F) \leqq 1$.

Soient $X$ un ensemble de combinaisons $(\not \equiv 0)$ linéaires de $f_{0}, \cdots, f_{n}$, homogènes à coefficients constants et linéairement indépendantes $n+1$

Received February 4, 1976. 
à $n+1$ et $\lambda$ le nombre maximum de relations linéaires, homogènes à coefficients constants et indépendantes sur $C$ entre les fonctions $f_{0}, \cdots$, $f_{n}$. Ici, $C$ signifie le corps de nombre complexe. On note que $0 \leqq \lambda \leqq$ $n-1$.

On cherche quelques relations entre le nombre de combinaisons exceptionnelles dans $X$ et le nombre " $\lambda$ ".

D'après le théorème fondamental de Cartan ([1]), on sait que s'il y a $n+2$ combinaisons $F_{1}, \cdots, F_{n+1}, G$ dans $X$ telles que

$$
\delta(G)+\sum_{i=1}^{n+1} \delta\left(F_{i}\right)>n+1
$$

alors, $\lambda \geqq 1$ (voir Lemme 2). Récemment, on a démontré que, si $n-1$ combinaisons quelconque dans $\left\{F_{i}\right\}_{i=1}^{n+1}$ sont linéairement indépendantes de plus, alors $\lambda=1$ et il y a une dans $\left\{F_{i}\right\}_{i=1}^{n+1}$ qui est proportionnelle à $G$ ([8], Th. B'). C'est une réponse positive pour la conjecture d'Ozawa ([5]) et de Noguchi ([4]).

Dans ce mémoire, on démontre

"S'il y a $n+\mu+1$ combinaisons $F_{1}, \cdots, F_{n+1}, G_{1}, \cdots, G_{\mu}(1 \leqq \mu \leqq n-1)$ dans $X$ telles que

$$
\delta\left(G_{j}\right)+\sum_{i=1}^{n+1} \delta\left(F_{i}\right)>n+1 \quad(j=1, \cdots, \mu),
$$

alors $\lambda \geqq \mu . "$ (voir Lemme 8 ), et, en utilisant cela, on généralise les Théorèmes $B^{\prime}$ et $D^{\prime}$ dans [8]. La généralisation du Théorème $\mathrm{D}^{\prime}$ (voir Th. 2) est peut-être un point final parti d'un théorème de Niino et Ozawa ([3], Th. 3).

On utilise les symboles usuels de la théorie de Nevanlinna ([2]).

\section{Préliminaires}

1. Soient $f, X$ et $\lambda$ comme dans l'introduction. D'abord, on note que le nombre maximum de relations linéaires, homogènes à coefficients constants et linéairement indépendantes sur $C$ entre $n+1$ combinaisons quelconque de $X$ est égal à $\lambda$. Soient $F_{1}, \cdots, F_{n+1} n+1$ combinaisons quelconque dans $X$, alors il y a $n+1-\lambda$ combinaisons linéairement indépendantes sur $C$ dans $\left\{F_{i}\right\}_{i=1}^{n+1}$ (soient $g_{1}, \cdots, g_{n+1-2}$ ) telles que tous les éléments dans $X$ sont représentés par $g_{1}, \ldots, g_{n+1-2}$ à coefficients constants : 


$$
F=\alpha_{F^{1}} g_{1}+\cdots+\alpha_{F n+1-\lambda} g_{n+1-\lambda} \quad\left(F \in X, \alpha_{F^{i}} \in C\right) .
$$

Evidemment, $g=\left(g_{1}, \cdots, g_{n+1-\lambda}\right)$ est un système transcendant dans $|z|<\infty$. On dit que telles $g_{1}, \cdots, g_{n+1-\lambda}$ forment une base de $X$.

On donne quelques lemmes qui seront utilisés après.

LEMME 1. Soient $H_{1}, \cdots, H_{k}(2 \leqq k \leqq n+1) k$ combinaisons quelconque dans $X$. Alors,

$$
m\left(r,\left\|H_{1}, \cdots, H_{k}\right\| / H_{1} \cdots H_{k}\right)=O(\log r T(r, f))(r \rightarrow \infty, r \notin E),
$$

où $\left\|H_{1}, \cdots, H_{k}\right\|$ signifie le wronskian de $H_{1}, \cdots, H_{k}$ et $E$ est un ensemble de $r$ de mesure linéaire finie.

Une démonstration de ce lemme est contenue dans celle du théorème fondamental de Cartan ([1]).

LEMME 2. Quand $\lambda=0, \sum_{F \in X} \delta(F) \leqq n+1 \quad$ ([1]).

LEMme 3. $|T(r, f)-T(r, g)|<O(1)$.

C'est trivial d'après les définitions de $T(r, f), T(r, g)$ et $g$.

LEMme 4. S'il y a $\lambda+1$ combinaisons dans $X$ tous les rapports entre lesquelles sont constants, on a

$$
\sum_{F \in X} \delta(F) \leqq n+\lambda+1 \quad([6], \text { Th. } 6) .
$$

LEMME 5. S'il y a $2 n$ combinaisons $F_{1}, \cdots, F_{2 n}$ dans $X$ telles que

$$
\delta\left(F_{n+1+j}\right)+\sum_{i=1}^{n+1} \delta\left(F_{i}\right)>n+1 \quad(j=1, \cdots, n-1)
$$

et si $\lambda=n-1$, alors les combinaisons $F_{1}, \cdots, F_{2 n}$ se repartissent en deux classes jouissant les propriétés suivantes:

1) Chaque classe contient $n$ combinaisons.

2) Tous les rapports entre deux éléments quelconque dans une même classe sont des constantes. ([8], Lemme 6).

2. Soit $X^{0}$ un sous-ensemble quelconque de $X-\left\{g_{1}, \cdots, g_{n+1-\lambda}\right\}$. On introduit une relation equivalente dans $X^{0}$.

DÉFINITION 1. i) $X^{0} \ni H_{1}, H_{2}, H_{j}=\sum_{i=1}^{n+1-\lambda} \alpha_{j i} g_{i}(j=1,2)$, $H_{1} \approx H_{2} \Leftrightarrow$ il y a un $i_{0}$ telque $\alpha_{1 i_{0}} \neq 0, \alpha_{2 i_{0}} \neq 0$. 
ii) $X^{0} \ni F, G$,

$F \sim G \Leftrightarrow F \approx G$, ou il existent $H_{1}, \cdots, H_{s}$ dans $X^{0}$ telles que $F \approx H_{1}$, $H_{1} \approx H_{2}, \cdots, H_{s} \approx G$.

Proposition 1. La relation “ " est une relation équivalente dans $X^{0}$

C'est trivial par définition.

On classifie $X^{0}$ par cette relation équivalente. Soit

$$
X^{0} / \sim=\left\{X_{1}^{0}, \cdots, X_{p}^{0}\right\} \quad(1 \leqq p \leqq n+1-\lambda) .
$$

DÉFINITION 2.

$A_{\ell}=\left\{g_{i} ;\right.$ il y a au moins une $F$ dans $X_{\ell}^{0}$ telle que $\left.\alpha_{F i} \neq 0\right\}$,

$A_{0}=\left\{g_{i}\right\}_{i=1}^{n+1-2}-\bigcup_{\ell=1}^{p} A_{\ell}$,

$\nu_{\ell}=$ le nombre d'éléments dans $A_{\ell}(\ell=0,1, \cdots, p)$.

Par définition, on obtient, tout de suite, la proposition suivante.

Proposition 2. i) $A_{\ell_{1}} \cap A_{\ell_{2}}=\phi\left(\ell_{1} \neq \ell_{2}\right)$.

ii) $\sum_{\ell=0}^{p} \nu_{\ell}=n+1-\lambda$.

\section{Lemme fondamental}

Soient $f, X$ et $\lambda$ comme précédent. Maintenant supposons qu'il y ait $n+\mu+1(1 \leqq \mu \leqq n-1)$ combinations $F_{1}, \cdots, F_{n+\mu+1}$ dans $X$ telles que

$$
\delta\left(F_{n+1+j}\right)+\sum_{i=1}^{n+1} \delta\left(F_{i}\right)>n+1 \quad(j=1, \cdots, \mu) .
$$

On note que $\lambda \geqq 1$ d'après le Lemme 2 . On peut supposer que $F_{1}, \cdots$, $F_{n+1-\lambda}$ forment une base de $X$. On utilise $\left\{F_{1}, \cdots, F_{n+1-\lambda}\right\}$ au lieu de $\left\{g_{1}, \cdots, g_{n+1-\lambda}\right\}$ dans $\S 2$. Représentons $F_{n+2-\lambda}, \cdots, F_{n+\mu+1}$ par $F_{1}, \cdots$, $F_{n+1-\lambda}$ :

$$
F_{n+1-\lambda+k}=\alpha_{k 1} F_{1}+\cdots+\alpha_{k n+1-\lambda} F_{n+1-\lambda} \quad(k=1, \cdots, \lambda+\mu)
$$

Proposition 3. Pour chaque $k$, il y a au moins un coefficient qui est égal à zéro.

Démonstration. Si tous les coefficient $\alpha_{k 1}, \cdots, \alpha_{k n+1-\lambda}$ sont différents de zéro, on peut appliquer le Lemme 2 à $\tilde{F}=\left(F_{1}, \cdots, F_{n+1-2}\right)$ et $\tilde{X}=$ 
$\left\{F_{1}, \cdots, F_{n+1-\lambda}, F_{n+1-\lambda+k}\right\}$. On obtient l'inégalité

$$
\delta\left(F_{n+1-\lambda+k}\right)+\sum_{i=1}^{n+1-\lambda} \delta\left(F_{i}\right) \leqq n+1-\lambda
$$

d'après le Lemme 3 . C'est contraire à (1).

Ici, on applique la discussion dans $\S 2-2$ à $Y^{0}=\left\{F_{n+1-\lambda+k}\right\}_{k=1}^{2}$ et $Y^{j}=$ $Y^{0} \cup\left\{F_{n+1+i}\right\}(j=1, \cdots, \mu)$ en utilisant $\left\{F_{1}, \cdots, F_{n+1-\lambda}\right\}$ au lieu de $\left\{g_{1}, \cdots\right.$, $\left.g_{n+1-\lambda}\right\}$. Soient

$$
\begin{array}{cl}
Y^{j} / \sim=\left\{Y_{1}^{j}, \cdots, Y_{p_{j}}^{j}\right\} & (j=0, \cdots, \mu), \\
B_{\ell}^{j}=\left\{F_{i} ; Y_{\ell}^{j} \ni F \text { telle que } \alpha_{F i} \neq 0\right\} & \left(\ell=1, \cdots, p_{j} ; j=0, \cdots, \mu\right), \\
B_{0}^{j}=\left\{F_{i}\right\}_{0=1}^{n+1-\lambda}-\bigcup_{\ell=1}^{P_{j}} B_{\ell}^{j} &
\end{array}
$$

où $\alpha_{F i}$ est le coefficient de $F_{i}(1 \leqq i \leqq n+1-\lambda)$ quand on représente $F$ par $F_{1}, \cdots, F_{n+1-\lambda}$.

De plus, soient

$\nu_{\ell}^{j}=$ le nombre d'éléments dans $B_{\ell}^{j}\left(\ell=0, \cdots, p_{j} ; j=0, \cdots, \mu\right)$.

D'après la Proposition 2, on a

$$
B_{\ell_{1}}^{j} \cap B_{\ell_{2}}^{j}=\phi \quad\left(\ell_{1} \neq \ell_{2}\right)
$$

et

$$
\sum_{\ell=0}^{p_{j}} \nu_{\ell}^{j}=n+1-\lambda \quad(j=1, \cdots, \mu) .
$$

LEMME 6. $\nu_{\ell}^{j} \leqq n-\lambda\left(\ell=0, \cdots, p_{j} ; j=0, \cdots, \mu\right)$.

Démonstration. Par définition,

$$
\nu_{0}^{j} \leqq n-\lambda
$$

pour chaque $j$. On démontre quand $j=1$. Supposons qu'il y ait un $\ell(\geqq 1)$ tel que

$$
\nu_{\ell}^{1}>n-\lambda .
$$

Alors, $p_{1}=1, Y_{1}^{1}=Y^{1}, B_{1}^{1}=\left\{F_{1}, \cdots, F_{n+1-\lambda}\right\}$ et $B_{0}^{1}=\phi . \quad$ Par conséquent, $\ell=1, \nu_{1}^{1}=n+1-\lambda$. On utilise les représentations (2). Dans ce cas, pour simplifier, on met

$$
\begin{gathered}
H_{k}=F_{n+1-\lambda+k} \quad(k=1, \cdots, \lambda+1), \\
H_{k}=\alpha_{k 1} F_{1}+\cdots+\alpha_{k n+1-\lambda} F_{n+1-\lambda} \quad(k=1, \cdots, \lambda+1) .
\end{gathered}
$$


On note que, grâce à la Proposition 3 , il y a au moins un coefficient égal à zéro pour chaque $k$.

I. Comme $\nu_{1}^{1}=n+1-\lambda$, il y a au moins une combinaison dans $\left\{H_{1}, \cdots, H_{2+1}\right\}$ telle que le nombre de coefficients différents de zéro à (2) est au moins deux. On peut supposer que $H_{1}$ est telle combinaison.

Soient

$$
\begin{gathered}
\alpha_{1 i(1)_{1}} \neq 0, \cdots, \alpha_{1 i(1) m_{1}} \neq 0 \\
\alpha_{1 i}=0 \quad\left(i \neq i(1)_{1}, \cdots, i(1)_{m_{1}}\right) \quad\left(2 \leqq m_{1} \leqq n-\lambda\right) .
\end{gathered}
$$

C'est-à-dire,

$$
H_{1}=\alpha_{1 i(1) 1} F_{i(1)_{1}}+\cdots+\alpha_{1 i(1)_{1}} F_{i(1)_{m_{1}}}
$$

Alors, de (4), on obtient

$$
\alpha_{1 i(1) m} F_{i(1) m}=H_{1} \Delta_{1}^{m} / \Delta_{1} \quad\left(m=1, \cdots, m_{1}\right)
$$

où

$$
\Delta_{1}=\left\|F_{i(1)_{1}}, \cdots, F_{i(1)_{1}}\right\| / F_{i(1)_{1}} \cdots F_{i(1)_{m_{1}}}
$$

et

$$
\Delta_{1}^{m}=\frac{\left\|F_{i(1)}, \cdots, F_{i(1) m-1}, H_{1}, F_{i(1)_{m+1}}, \cdots, F_{i(1)_{m}}\right\|}{F_{i(1)_{1}} \cdots F_{i(1)_{m-1}} H_{1} F_{i(1)_{m+1}} \cdots F_{i(1)_{m_{1}}}} .
$$

De (5), on a l'inégalité

$$
\max _{\alpha_{1 i} \neq 0} \log \left|F_{i}\right| \leqq \log \left|H_{1}\right|+\log ^{+}\left|\frac{1}{\Delta_{1}}\right|+\sum_{m=1}^{m_{1}} \log ^{+}\left|\Delta_{1}^{m}\right|+\mathrm{O}(1)
$$

II. Parce que

$$
\left\{F_{i} ; \alpha_{1 i} \neq 0\right\} \subsetneq\left\{F_{1}, \cdots, F_{n+1-\lambda}\right\}
$$

et

$$
\nu_{1}^{1}=n+1-\lambda,
$$

il y a une combinaison $H_{k}$ dans $\left\{H_{2}, \cdots, H_{\lambda+1}\right\}$ et un $i$ tels que

$$
H_{1} \approx H_{k}, \quad \alpha_{1 i}=0 \text { et } \alpha_{k i} \neq 0 \text {. }
$$

On peut supposer que $H_{k}=H_{2}$.

Soient 


$$
\begin{gathered}
\left\{i ; \alpha_{1 i}=0, \alpha_{2 i} \neq 0\right\}=\left\{i(2)_{1}, \cdots, i(2)_{m_{2}}\right\} \\
\Delta_{2}=\left\|H_{2}, F_{i(2) 1}, \cdots, F_{i(2)_{2}}\right\| / H_{2} F_{i(2)} \cdots F_{i(2)_{m_{2}}}
\end{gathered}
$$

et

$$
\Delta_{2}^{m}=\frac{\left\|H_{2}, F_{i(2) 1}, \cdots, F_{i(2) m-1}, \tilde{F}_{2}, F_{i(2) m+1}, \cdots, F_{i(2) m_{2}}\right\|}{H_{2} F_{i(2) 1} \cdots F_{i(2) m-1} \tilde{F}_{2} F_{i(2)_{m+1}} \cdots F_{i(1)_{m}}}
$$

où

$$
\tilde{F}_{2} \equiv-\sum_{\substack{\alpha_{1 i} \neq 0 \\ \alpha_{2} i \neq 0}} \alpha_{2 i} F_{i}=-H_{2}+\sum_{\substack{\alpha_{2} \neq 0 \\ \alpha_{1 i}=0}} \alpha_{2 i} F_{i}
$$

Parce que $H_{1} \approx H_{2}$ et $\left\{i ; \alpha_{1 i} \neq 0, \alpha_{2 i} \neq 0\right\} \neq \phi$,

$$
\tilde{F}_{2} \not \equiv 0 \text {. }
$$

Alors, on obtient

$$
\alpha_{2 i(2) m}=\tilde{F}_{2} \Delta_{2}^{m} / \Delta_{2} \quad\left(m=1, \cdots m_{2}\right) .
$$

De (8), on a l'inégalité

$$
\max _{\substack{\alpha_{2 i} \neq 0 \\ \alpha_{1}=0}} \log \left|F_{i}\right| \leqq \log \left|\tilde{F}_{2}\right|+\log ^{+}\left|\frac{1}{\Delta_{2}}\right|+\sum_{m=1}^{m_{2}} \log ^{+}\left|\Delta_{2}^{m}\right|+O(1)
$$

et de (7),

$$
\log \left|\tilde{F}_{2}\right| \leqq \max _{\substack{\alpha_{1 i} \neq 0 \\ \alpha_{2 i} \neq 0}} \log \left|F_{i}\right|+0(1) \leqq \max _{\alpha_{1 i} \neq 0} \log \left|F_{i}\right|+O(1)
$$

De plus, on a

$$
m_{1}+m_{2} \leqq n-\lambda \text {. }
$$

En effet, si $m_{1}+m_{2}=n+1-\lambda$, de (6), (9) et (10), on a

$$
\begin{aligned}
\max _{1 \leqq i \leqq n+1-i} \log \left|F_{i}\right| \leqq & \log \left|H_{1}\right|+\log ^{+}\left|\frac{1}{\Delta_{1}}\right|+\log ^{+}\left|\frac{1}{\Delta_{2}}\right|+\sum_{m=1}^{m_{1}} \log ^{+}\left|\Delta_{1}^{m}\right| \\
& +\sum_{m=1}^{m_{2}} \log ^{+}\left|\Delta_{2}^{m}\right|+O(1),
\end{aligned}
$$

de sorte qu'en intégrant à régard de $\theta$, on obtient l'inégalité suivante d'après les Lemmes 1 et 3 :

$$
\begin{aligned}
T(r, f) \leqq & N\left(r, 0, H_{1}\right)+m\left(r, 1 / \Delta_{1}\right)+m\left(r, 1 / \Delta_{2}\right)+\sum_{m=1}^{m_{1}} m\left(r, \Delta_{1}^{m}\right) \\
& +\sum_{m=1}^{m_{2}} m\left(r, \Delta_{2}^{m}\right)+O(1)
\end{aligned}
$$




$$
\leqq \sum_{i=1}^{n+3-\lambda} N\left(r, 0, F_{i}\right)+S(r)
$$

où $S(r)=O(\log r T(r, f))(r \rightarrow \infty, r \notin E)$.

Parce que $H_{k}=F_{n+1-\lambda+k}$,

$$
\begin{gathered}
m\left(r, 1 / \Delta_{k}\right)=N\left(r, \Delta_{k}\right)+m\left(r, \Delta_{k}\right)-N\left(r, 1 / \Delta_{k}\right)+0(1), \quad(k=1,2) \\
N\left(r, \Delta_{1}\right) \leqq \sum_{m=1}^{m_{k}} N\left(r, 0, F_{i(k) m}\right) \\
N\left(r, \Delta_{2}\right) \leqq \sum_{m=1}^{m_{2}} N\left(r, 0, F_{i(2) m}\right)+N\left(r, 0, H_{2}\right)
\end{gathered}
$$

et

$$
\left\{F_{i(1) m}\right\}_{m=1}^{m_{1}} \cup\left\{F_{i(2) m}\right\}_{m=1}^{m_{2}}=\left\{F_{i}\right\}_{i=1}^{n+1-\lambda}
$$

En conséquence, on a

$$
\sum_{i=1}^{n+3-\lambda} \delta\left(F_{i}\right) \leqq n+2-\lambda,
$$

qui est contraire à (1). Cela veut dire que

$$
\left\{F_{i} ; \alpha_{1 i} \neq 0 \text { ou } \alpha_{2 i} \neq 0\right\} \subsetneq\left\{F_{i}\right\}_{i=1}^{n+1-\lambda} .
$$

III. Comme $\nu_{1}^{1}=n+1-\lambda$, il y a au moins une $H_{j}$ dans $\left\{H_{3}, \cdots, H_{\lambda+1}\right\}$ et un $i(1 \leqq i \leqq n+1-\lambda)$ tels que

$$
H_{1} \approx H_{j} \text { ou } H_{2} \approx H_{j}
$$

et

$$
\alpha_{1 i}=0, \quad \alpha_{2 i}=0, \quad \alpha_{j i} \neq 0 .
$$

On peut supposer que $H_{j}=H_{3}$. Soient

$$
\begin{gathered}
\left\{i ; \alpha_{1 i}=0, \alpha_{2 i}=0, \alpha_{3 i} \neq 0\right\}=\left\{i(3)_{1}, \cdots, i(3)_{m_{3}}\right\}, \\
\Delta_{3}=\left\|H_{3}, F_{i(3)_{1}}, \cdots, F_{i(3)_{3}}\right\| / H_{3} F_{i(3)_{1}} \cdots F_{i(3)_{m_{3}}}, \\
\Delta_{3}^{m}=\frac{\left\|H_{3}, F_{i(3)_{1}}, \cdots, F_{i(3)_{m-1}}, \tilde{F}_{3}, F_{i(3)_{m+1}}, \cdots, F_{i(3)_{3}}\right\|}{H_{3} F_{i(3)_{1}} \cdots F_{i(3)_{m-1}} \tilde{F}_{3} F_{i(3)_{m+1}} \cdots F_{i(3)_{m_{3}}}}
\end{gathered}
$$

où

$$
\tilde{F}_{3}=-\sum_{\substack{\alpha_{i j} \neq 0, \alpha_{1 i} \neq 0 \text { ou } \alpha_{2 i} \neq 0}} \alpha_{3 i} F_{i}=-H_{3}+\sum_{\substack{\alpha_{3 i} \neq 0 \\ \alpha_{2 i}=0 \\ \alpha_{3 i}=0}} \alpha_{3 i} F_{i} .
$$

Parce que $H_{1} \approx H_{3}$ ou $H_{2} \approx H_{3}$, 
$\left\{i ; \alpha_{3 i} \neq 0,\left|\alpha_{1 i}\right|+\left|\alpha_{2 i}\right| \neq 0\right\} \neq \phi$ et $\quad \tilde{F}_{3} \neq \equiv 0$.

Alors, on a

$$
\alpha_{3 i(3) m} F_{i(3) m}=\tilde{F}_{3} \Delta_{3}^{m} / \Delta_{3} \quad\left(m=1, \cdots, m_{3}\right)
$$

et de cela, on a

$$
\max _{\substack{\alpha_{3 i} \neq 0, \alpha_{2} i=0 \\ \alpha_{1 i}=0}} \log \left|F_{i}\right| \leqq \log \left|\tilde{F}_{3}\right|+\log ^{+}\left|\frac{1}{\Delta_{3}}\right|+\sum_{m=1}^{m_{3}} \log ^{+}\left|\Delta_{3}^{m}\right|+O(1)
$$

et

$$
\log \left|\tilde{F}_{3}\right| \leqq \max _{\alpha_{1 i} \neq 0 \text { ou } \alpha_{2 i} \neq 0} \log \left|F_{i}\right|+O(1)
$$

Des inégalités (6), (9), (10), (12) et (13), on a

$$
\max _{\substack{\alpha_{1} \neq 0 \text { ou } \alpha_{2 i} \neq 0 \\ \text { ou } \alpha_{3 i} \neq 0}} \log \left|F_{i}\right| \leqq \log \left|H_{1}\right|+\sum_{k=1}^{3} \log ^{+}\left|\frac{1}{\Delta_{k}}\right|+\sum_{k=1}^{3} \sum_{m=1}^{m_{k}} \log ^{+}\left|\Delta_{k}^{m}\right|+O(1) \text {. }
$$

En utilisant cette inégalité, d'après l'hypothèse (1), on a

$$
m_{1}+m_{2}+m_{3} \leqq n-\lambda
$$

comme dans II, (8). C'est-à-dire,

$$
\left\{F_{i} ; \alpha_{1 i} \neq 0 \text { ou } \alpha_{2 i} \neq 0 \text { ou } \alpha_{3 i} \neq 0\right\} \subseteq\left\{F_{i}\right\}_{i=1}^{n+1-\lambda} \text {. }
$$

De la même manière, on obtient

$\left\{F_{i} ; \alpha_{1 i} \neq 0\right.$ ou $\cdots$ ou $\left.\alpha_{q i} \neq 0\right\} \subsetneq\left\{F_{i}\right\}_{i=1}^{n+1-\lambda} \quad(q=1,2, \cdots, \lambda+1)$.

IV. D'autre part, comme

$$
\begin{gathered}
\left\{\boldsymbol{F}_{i} ; \alpha_{1 i} \neq 0\right\} \subseteq\left\{\boldsymbol{F}_{i} ; \alpha_{1 i} \neq 0 \text { ou } \alpha_{2 i} \neq 0\right\} \subsetneq \cdots \subsetneq\left\{\boldsymbol{F}_{i} ; \alpha_{1 i} \neq 0 \text { ou } \cdots\right. \\
\text { ou } \left.\alpha_{q i} \neq 0\right\} \subsetneq \cdots \subset\left\{\boldsymbol{F}_{i}\right\}_{i=1}^{n+1-\lambda}
\end{gathered}
$$

et l'ensemble $\left\{F_{i}\right\}_{i=1}^{n+1-\lambda}$ est fini, il faut qu'il y ait un $t(\leqq \lambda+1)$ tel que

$$
\left\{F_{i} ; \alpha_{1 i} \neq 0 \text { ou } \cdots \text { ou } \alpha_{t i} \neq 0\right\}=\left\{F_{i}\right\}_{i=1}^{n+1-\lambda} \text {. }
$$

Dans ce cas, comme (14), on obtient l'inégalité

$$
\begin{aligned}
\max _{\substack{\alpha_{1 i} \neq \text { ou } \ldots \ldots . . \\
\text { ou } \alpha_{t i} \neq 0}} \log \left|F_{i}\right| & =\max _{\substack{1 \leqq i \leqq n+1-1 \\
1}} \log \left|F_{i}\right| \\
& \leqq \log \left|H_{1}\right|+\sum_{k=1}^{t} \log ^{+}\left|\frac{1}{\Delta_{k}}\right|+\sum_{k=1}^{t} \sum_{m=1}^{m_{k}} \log ^{+}\left|\Delta_{k}^{m}\right|+O(1) .
\end{aligned}
$$


De cette inégalité, on a, comme dans II,

$$
T(r, f) \leqq \sum_{i=1}^{n+1-\lambda+t} N\left(r, 0, F_{i}\right)+S(r),
$$

où $S(r)=O(\log r T(r, f))(r \rightarrow \infty, r \notin E)$.

Par conséquent, on obtient l'inégalité

$$
\sum_{i=1}^{n+1-\lambda+t} \delta\left(F_{i}\right) \leqq n+t-\lambda,
$$

qui est contraire à (1). Cela veut dire qu'il faut

$$
\nu_{\ell}^{1} \leqq n-\lambda
$$

pour $\ell=1, \cdots, p_{1}$.

De la même manière, on obtient

$$
\nu_{\ell}^{j} \leqq n-\lambda
$$

pour $\ell=1, \cdots, p_{j}, j=2, \cdots, \mu$.

On peut supposer que $F_{n+1+j}$ appartiennent à $Y_{1}^{j}$ sans restriction de généralité $(j=1, \cdots, \mu)$.

LEMME 7. i) Quand $\nu_{0}^{0}=0$, pour chaque $j(=1, \cdots, \mu)$, il existent un $\ell(j, 1)$ tel que

$$
B_{\ell(j, 1)}^{0} \subset B_{1}^{j}
$$

et un $\ell(j, 2)$ tel que

$$
B_{\ell(j, 2)}^{0} \cap B_{1}^{j}=\phi .
$$

ii) Quand $\nu_{0}^{0}>0$,

$$
B_{0}^{0} \subset B_{1}^{j}
$$

et il a un $\ell(j)$ tel que

$$
B_{\ell(j)}^{0} \cap B_{1}^{j}=\phi \quad(j=1, \cdots, \mu) .
$$

Démonstration. i) Comme

$$
\sum_{\ell=1}^{p_{0}} \nu_{\ell}^{0}=n+1-\lambda,
$$

il y a au moins un $\ell(j, 1)$ tel que

$$
B_{\ell(j, 1)}^{0} \cap B_{1}^{j} \neq \phi .
$$


Alors, par définition,

$$
Y_{\ell(j, 1)}^{0} \subset Y_{1}^{j}
$$

et on a

$$
B_{\ell(j, 1)}^{0} \subset B_{1}^{j}
$$

Et puis, si

$$
B_{\ell}^{0} \subset B_{1}^{j}
$$

pour $\ell=1, \cdots, p_{j}$, alors

$$
\nu_{1}^{j}=n+1-\lambda .
$$

C'est contraire au Lemme 6. Cela veut dire qu'il y a un $\ell(j, 2)$ tel que

$$
B_{\ell(j, 2)}^{0} \cap B_{1}^{j}=\phi \text {. }
$$

ii) On peut supposer que $B_{0}^{0}=\left\{F_{1}, \cdots, F_{v_{0}}\right\}$. Pour tous les éléments dans $\bigcup_{\ell=0}^{p_{0}} Y_{\ell}^{0}$, les coefficients de $F_{1}, \cdots, F_{\nu_{0}^{0}}$ sont tous égals à zéro par définition. En conséquence quand on représente $F_{n+1+j}$ par $F_{1}, \ldots, F_{n+1-\lambda}$, les coeffcients de $F_{1}, \cdots, F_{\nu_{0}^{0}}$ sont différents de zéro. En effet, s'il y en a un qui est égal à zéro (soit le coefficient de $F_{1}$ égal à zéro, par exemple), le nombre de relations linéaires, homogènes à coefficients constants et indépendantes entre $n+1$ combinaisons $F_{2}, \ldots, F_{n+1}, F_{n+1+j}$ devient $\lambda+1$, qui est contraire à la définition de $\lambda$. Cela veut dire que

$$
B_{0}^{0} \subset B_{1}^{j} \text {. }
$$

$\mathrm{Si}$, pour chaque $\ell\left(=1, \cdots, p_{0}\right)$,

$$
B_{\ell}^{0} \cap B_{1}^{j} \neq \phi,
$$

alors,

$$
Y_{\ell}^{0} \subset Y_{1}^{j}
$$

et

$$
B_{\ell}^{0} \subset B_{1}^{j}
$$

Par conséquent,

$$
\bigcup_{\ell=0}^{p_{0}} B_{\ell}^{0} \subset B_{1}^{j} \quad \text { et } \quad \nu_{1}^{j}=n+1-\lambda
$$


qui est contraire au Lemme 6. C'est-à-dire, il y a un $\ell(j)$ tel que

$$
B_{\ell(j)}^{0} \cap B_{1}^{j}=\phi .
$$

LEMME 8. S'il y a $n+\mu+1$ combinaisons $\left\{F_{1}, \cdots, F_{n+\mu+1}\right\}(1 \leqq \mu \leqq$ $n-1)$ dans $X$ telles que

$$
\delta\left(F_{n+1+j}\right)+\sum_{i=1}^{n+1} \delta\left(F_{i}\right)>n+1 \quad(j=1, \cdots, \mu),
$$

alors, $\lambda \geqq \mu$.

Démonstration. On applique le Lemme 7 au

$$
Y^{j}=\left\{F_{1}, \cdots, F_{n+1}, F_{n+1+j}\right\} \quad(j=1, \cdots, \mu) .
$$

C'est possible d'après l'hypothèse (1). Pour chaque $j$, il y a au moins une classe $B_{\ell(j)}^{0}$ telle que, quand on représente $F_{n+1+j}$ par $F_{1}, \cdots, F_{n+1-\lambda}$, tous les coefficients d'éléments dans $B_{\ell(j)}^{0}$ sont égals à zéro.

1) Le cas $\nu_{0}^{0}=0$. Quand on représente $F_{n+1-\lambda+k}(k=1, \cdots, \lambda)$ par $F_{1}, \cdots, F_{n+1-\lambda}$, pour chaque $k$, il y a $p_{0}-1$ classes $B_{\ell(k) 1}^{0}, \cdots, B_{\ell(k)_{p_{0}-1}}^{0}$ telles que tous les coefficients d'éléments dans $\bigcup_{m=1}^{p_{0}-1} B_{\ell(k) m}^{0}$ sont égal à zéro. Par conséquent, en considérant que le nombre maximum de relations linéaires, homogènes à coefficients constants et linéairement indépendantes entre $n+1$ éléments quelconque dans $X$ est $\lambda$, il faut que

$$
\mu+\left(p_{0}-1\right) \lambda \leqq p_{0} \lambda .
$$

C'est-à-dire, on a

$$
\mu \leqq \lambda
$$

2) Le cas $\nu_{0}^{0}>0$. Quand on représente $F_{n+1-\lambda+k}(k=1, \cdots, \lambda)$ par $F_{1}, \cdots, F_{n+1-\lambda}$, il y a $p_{0}$ classes $B_{0}^{0}, B_{\ell(k) 1}^{0}, \cdots, B_{\ell(k) p_{0}-1}^{0}$ telles que tous les coefficients d'éléments dans $B_{0}^{0} \cup\left(\bigcup_{m=1}^{p_{0}-1} B_{\ell(k) m}^{0}\right)$ sont égals à zéro pour chaque $k$. Par conséquent, comme 1) il faut que

$$
\mu+p_{0} \lambda \leqq\left(p_{0}+1\right) \lambda
$$

C'est-à-dire, on a

$$
\mu \leqq \lambda
$$




\section{Théorèmes}

Soient $f, X$ et $\lambda$ comme précédent. Dans ce paragraphe, on donne deux théorèmes et quelques applications.

THÉORÈME 1. S'il y $a n+\mu+1(1 \leqq \mu \leqq n-2)$ combinaisons $F_{1}, \cdots$, $F_{n+\mu+1}$ dans $X$ telles que

1) $n-\mu$ combinaisons quelconque dans $\left\{F_{i}\right\}_{i=1}^{n+1}$ sont linéairement indépendantes sur $C$ et

2) $\delta\left(F_{n+1+j}\right)+\sum_{i=1}^{n+1} \delta\left(F_{i}\right)>n+1(j=1, \cdots, \mu)$,

alors,

i) $\lambda=\mu$,

ii) il y a une combinaisons $F_{i_{0}}$ dans $\left\{F_{i}\right\}_{i=1}^{n+1}$ telle que $F_{i_{0}}, F_{n+2}, \cdots$, $F_{n+\mu+1}$ sont proportionnelles aux unes aux autres,

iii) pour $F$ quelconque dans $X-\left\{F_{1}, \cdots, F_{n+\mu+1}\right\}$,

$$
\delta(F)+\sum_{i=1}^{n+1} \delta\left(F_{i}\right) \leqq n+1
$$

iv) $\sum_{F \in X} \delta(F) \leqq n+\mu+1$.

Démonstration. On utilise les mêmes notations dans $\S 3$. i) D'après l'hypothèse 1 ), $\nu_{1}^{0} \geqq n-\mu$ et en vertu du Lemme $6, \nu_{1}^{0} \leqq n-\lambda$. Par conséquent, on a

$$
\mu \geqq \lambda
$$

D'autre part, grâce au Lemme 8 , hypothèse 2) entraîne que

$$
\lambda \geqq \mu \text {. }
$$

C'est-à-dire, on a $\lambda=\mu$.

ii) L'hypothèse 1) et i) entrainent que

$$
\nu_{1}^{0}=\nu_{2}^{0}=\cdots=\nu_{p_{0}}^{0}=n-\lambda .
$$

De plus, comme

$$
\sum_{\ell=0}^{p_{0}} \nu_{\ell}^{0}=n+1-\lambda
$$

il faut que

$$
p_{0}=1, \quad \nu_{1}^{0}=n-\lambda \quad \text { et } \quad \nu_{0}^{0}=1
$$


Soit $B_{0}^{0}=\left\{F_{i_{0}}\right\}$. Alors, en appliquant le Lemme 7 , ii), on a

$$
F_{n+1+j}=\alpha_{j} F_{i_{0}} \quad(j=1, \cdots, \mu) .
$$

iii) S'il y a une $F_{0}$ dans $X-\left\{F_{1}, \cdots, F_{n+\mu+1}\right\}$ telle que

$$
\delta\left(F_{0}\right)+\sum_{i=1}^{n+1} \delta\left(F_{i}\right)>n+1
$$

en considérant l'hypothèse 2 ), on a $\lambda \geqq \mu+1$ d'après le Lemme 8 . C'est contraire à i).

iv) D'après ii), en appliquant le Lemme 4, on a la conclusion tout de suite.

THÉORÈme 2. S'il y a $2 n$ combinaisons $\left\{F_{i}\right\}_{i=1}^{2 n}$ dans $X$ telles que

$$
\delta\left(F_{n+1+j}\right)+\sum_{i=1}^{n+1} \delta\left(F_{i}\right)>n+1 \quad(j=1, \cdots, n-1),
$$

alors, $\left\{F_{i}\right\}_{i=1}^{2 n}$ se repartissent en deux classes jouissants les propriétés suivantes:

i) Chaque classe contient $n$ combinaisons.

ii) Tous les rapports entre deux éléments quelconque dans une même classe sont des constantes.

Démonstration. $f$ étant trancendant, l'hypothèse entraîne que

$$
\lambda=n-1
$$

d'après le Lemme 8. Par conséquent, on a ce théorème tout de suite du Lemme 5 .

N.B. 1) $\mathrm{Au}$ théorème 1 , quand $\mu=1$, on a le Théorème $\mathrm{B}^{\prime}$ dans [8]. 2) Le Théorème 2 est une amélioration du Théorème $C^{\prime}$ dans [7].

Corollaire 1. i) S'il y a une combinaison exceptionnelle au sens de Picard (ou lacunaire) dans $\left\{F_{n+1+j}\right\}_{j=1}^{\mu}$ au Théorème 1 , il y en $a \mu+1$ dans $\left\{F_{i}\right\}_{i=1}^{n+\mu+1}$.

ii) S'il y a une combinaison exceptionnelle au sens de Picard (ou lacunaire) dans $\left\{F_{i}\right\}_{i=1}^{2 n}$ au Théorème 2 , il y en a n-1 en outre.

CoRollaIRE 2. Soient $F_{1}, \cdots, F_{n+\lambda+2} n+\lambda+2$ combinaisons quelconque dans $X$. Alors, on a

$$
\sum_{i=1}^{n+\lambda+2} \delta\left(F_{i}\right) \leqq n+\lambda+1
$$


En effet, si

$$
\sum_{i=1}^{n+\lambda+2} \delta\left(F_{i}\right)>n+\lambda+1
$$

on a

$$
\delta\left(F_{n+1+j}\right)+\sum_{i=1}^{n+1} \delta\left(F_{i}\right)>n+1 \quad(j=1, \cdots, \lambda+1) .
$$

Cela veut dire que $\lambda \geqq \lambda+1$ d'après le Lemme 8 , qui est absurde.

CoRollaIRE 3. Il y a au plus $n+\lambda+1$ combinaisons exceptionnelles au sens de Picard dans $X$.

Finalement, on donne un exemple.

EXEMPLE. Soient $a_{1}, \cdots, a_{n+\mu+1}(1 \leqq \mu \leqq n-1) n+\mu+1$ valeurs complexes finies et distinctes. On met

$$
\begin{aligned}
F(z, w) & =f_{0} w^{n}+f_{1} w^{n-1}+\cdots+f_{n} \\
& =e^{z} \prod_{i=1}^{n}\left(w-a_{i}\right)+\prod_{j=0}^{\mu}\left(w-a_{n+1+j}\right) \sum_{k=1}^{n-1} A_{k} \frac{\prod_{m=1}^{n-\mu}\left(w-a_{m}\right)}{w-a_{k}}
\end{aligned}
$$

où

$$
A_{k}=z^{k-1} / \prod_{j=0}^{\mu}\left(a_{k}-a_{n+1+j}\right) \cdot \prod_{\substack{m=1 \\ m \neq k}}^{n-\mu}\left(a_{k}-a_{m}\right) \quad(k=1, \cdots, n-\mu) .
$$

Dans cette situation, soient

$$
\begin{gathered}
f=\left(f_{0}, \cdots, f_{n}\right), \quad X=\left\{w^{n} f_{0}+w^{n-1} f_{1}+\cdots+f_{n} ; w \in C\right\} . \\
F_{i} \equiv F\left(z, a_{i}\right) \quad(i=1, \cdots, n+\mu+1)
\end{gathered}
$$

Alors, $f$ est transcendant et $\left\{F_{i}\right\}_{i=1}^{n+\mu+1}$ satisfont les hypothèses $d u$ Théorème $1(\mu \leqq n-2)$ ou du Théorème $2(\mu=n-1)$.

\section{BIBLIOGRAPHIE}

[1] H. Cartan, Sur les zéros des combinaisons linéaires de $p$ fonctions holomorphes données. Mathematica, 7 (1933), 5-31.

[2] R. Nevanlinna, Le théorème de Picard-Borel et la théorie des fonctions méromorphes. Gauthier-Villars, Paris 1929.

[ 3 ] K. Niino et M. Ozawa, Deficiencies of an entire algebroid function. Kōdai Math. Sem. Rep., 22 (1970), 98-113.

[4] J. Noguchi, On the deficiencies and the existence of Picard exceptional values of 
entire algebroid function. Kodai Math. Sem. Rep., 26 (1974), 29-35.

[ 5 ] M. Ozawa, Deficiencies of an entire algebroid function III. Kōdai Math. Sem. Rep., 23 (1971), 486-492.

[ 6 ] N. Toda, Sur quelques combinaisons linéaires exceptionnelles au sens de Nevanlinna, Tôhoku Math. J., 23 (1971), 67-95.

[7] N. Toda, Sur quelques combinaisons linéaires exceptionnelles au sens de Nevanlinna, II. J. Math. Soc. Japan, 25 (1973), 158-167.

[8] N. Toda, Sur quelques combinaisons linéaires exceptionnelles au sens de Nevanlinna, IV. Nagoya Math. J., 59 (1975), 77-86.

Université de Nagoya 